\title{
How Much Anesthesia Research Has Academia in the United States Contributed in the Past 50 Years?
}

\author{
Alaa Abd-Elsayed, MD, MPH, Kenneth Fiala \\ Department of Anesthesiology, University of Wisconsin School of Medicine and Public Health, Madison, WI
}

We have noted an increase in regulations controlling research performed in the United States. We believe regulations have good intentions but cause performing research to be costly and difficult. In addition, we have noted that universities have more regulations than private practice settings because of the limited interaction with industry and availability of industrial funds, high overhead costs, high institutional taxes, and the amount of paperwork necessary for approvals.

As a result of these observations, we conducted several searches to determine how much published anesthesia research came from academic institutions (college, institution, school, or university) vs nonacademic institutions in the past 50 years (1968-2018). We believe that 50 years is a long enough period to draw conclusions about how much research was contributed from academic sources. Also, we predicted that analyzing beyond 50 years would lead to difficulty in finding academic affiliations. We used the Scopus database to conduct the searches. By using the advanced search tools in Scopus, we were able to narrow the results to the desired parameters.

We first searched for all published documents with the word "anesthesia" in the title, abstract, or as a keyword $(n=396,778)$ and then restricted the search to papers published in the last 50 years $(n=362,510)$.
We then searched for articles that had any affiliation with a college, school, institute, or university. To find results affiliated with universities or institutes that do not have English-language names, we used the wildcard searches "universit" and "instit"" to identify all results with those prefixes $(n=43,618,292)$. We then combined the searches of academic affiliation; presence of "anesthesia" in the title, abstract, or as a keyword; and publication in the last 50 years, and the result was 205,545 documents. Based on these search results, we concluded that $56.7 \%$ of anesthesia papers published worldwide in the past 50 years have come from academic sources (Figure 1).

The ability to search using the parameter of affiliation is why we used Scopus to retrieve data. However, one of the principal limitations we encountered is that the practice of listing all authors' affiliations has only recently begun. In the past, journals listed the affiliation of only the first author. Further, only one affiliation is commonly listed for each author even though an author can have multiple affiliations. Consequently, the number of papers from academia may be underrepresented in our analysis.

Because of this limitation, we conducted another search for documents with "anesthesia" in the title, abstract, or as a keyword, but we limited the results to the past 25 years (1993-2018). This search yielded 254,829 papers, and when

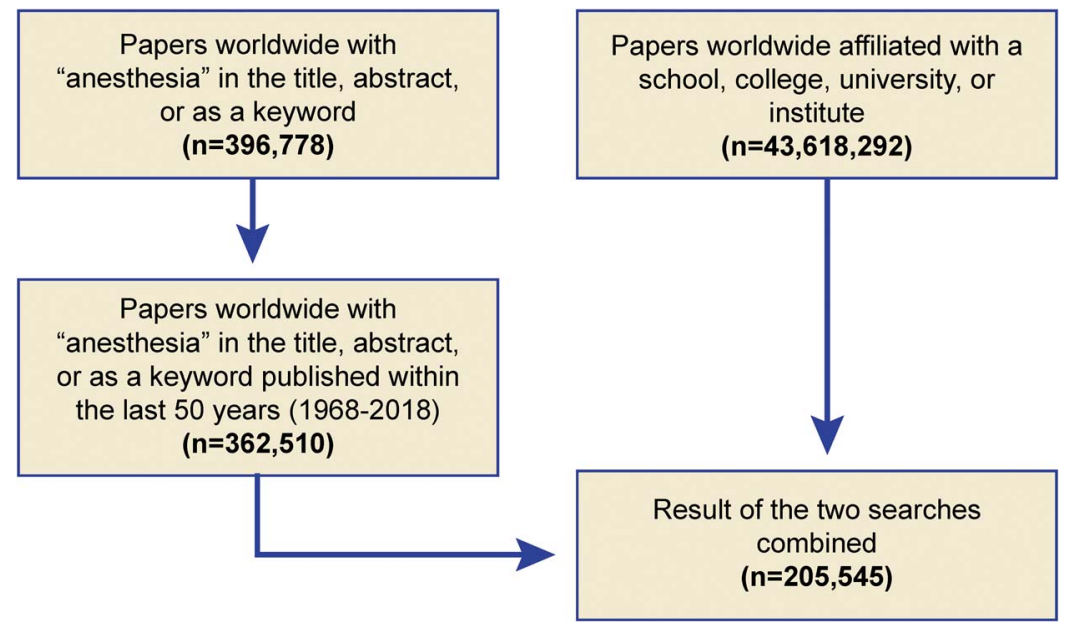

Figure 1. Results of worldwide searches for anesthesia-related papers and papers from academic sources. 


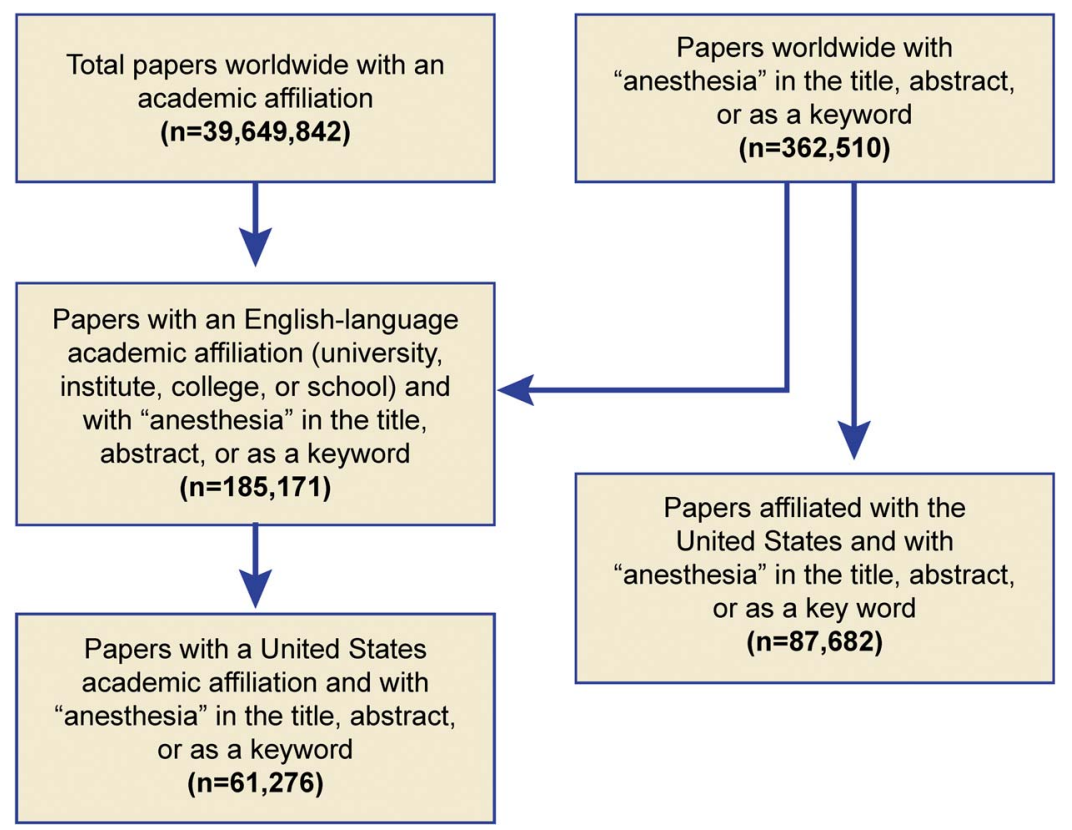

Figure 2. Results of worldwide and English-language/US delimited searches for anesthesia-related papers from academic sources in the past $\mathbf{5 0}$ years (1968-2018).

we added the delimiter of academic affiliation, the result was 169,995 papers. Based on these search results, $66.7 \%$ of anesthesia papers published worldwide in the past 25 years have come from academic sources, suggesting that the number of papers from academia could be underrepresented in the 50-year search results (56.7\%).

To determine the total number of papers with an Englishlanguage academic affiliation and published in the last 50 years, we limited the affiliation search by using the English spellings of "institute" and "university" ( $n=39,649,842)$. Refining the search to papers with "anesthesia" in the title, abstract, or as a keyword yielded 185,171 papers. Based on these results, we concluded that $51.1 \%$ of the 362,520 total anesthesia-related papers published in the past 50 years were from English-language academic sources.

Using the refining tool in Scopus, we chose the parameter of United States affiliation and narrowed the results to 61,276 documents. This result reflects all of the following parameters: presence of "anesthesia" in the title, abstract, or as a keyword; affiliation with an English-language academic source; affiliation with the United States; and publication in the past 50 years. Based on this search, $16.9 \%$ $(61,276 / 362,510)$ of anesthesia papers with academic affili- ations published worldwide in the past 50 years were from the United States (Figure 2).

A separate search returned 87,682 documents with "anesthesia" in the title, abstract, or as a keyword; with a US affiliation; and with a publication date in the last 50 years. This result suggests that $69.9 \%(61,276 / 87,682)$ of anesthesia documents published in the past 50 years were affiliated with a United States academic institution.

Our work shows that the United States contributed only $24.2 \%(87,682 / 362,510)$ of the anesthesia research worldwide in the last 50 years. Further, US academic institutions contributed an even smaller percentage (16.9\%) of the anesthesia research published in the past 50 years worldwide.

We need to examine the obstacles academic institutions encounter when performing research in the United States and work on solutions to increase our contribution to the worldwide literature. The obstacles may be related to extensive institutional review board (IRB) procedures, cost of IRB application, cost of coordinating and implementing studies, focus on clinical practice more than academia, lack of institutional support, legal liability, and other, yet-to-be-identified issues. 